\title{
Congruences for Fishburn numbers modulo prime powers
}

\author{
Armin Straub * \\ Department of Mathematics \\ University of Illinois at Urbana-Champaign
}

July 16, 2014

\begin{abstract}
The Fishburn numbers $\xi(n)$ are defined by the formal power series

$$
\sum_{n \geqslant 0} \xi(n) q^{n}=\sum_{n \geqslant 0} \prod_{j=1}^{n}\left(1-(1-q)^{j}\right) .
$$

Recently, G. Andrews and J. Sellers discovered congruences of the form $\xi(p m+j) \equiv 0$ modulo $p$, valid for all $m \geqslant 0$. These congruences have then been complemented and generalized to the case of $r$-Fishburn numbers by F. Garvan. In this note, we answer a question of Andrews and Sellers regarding an extension of these congruences to the case of prime powers. We show that, under a certain condition, all these congruences indeed extend to hold modulo prime powers.
\end{abstract}

\section{Introduction and main results}

The Fishburn numbers $\xi(n)$, which are defined by the formal power series

$$
\sum_{n \geqslant 0} \xi(n) q^{n}=\sum_{n \geqslant 0} \prod_{j=1}^{n}\left(1-(1-q)^{j}\right),
$$

count a variety of combinatorial objects, such as interval orders or unlabeled $(2+2)$-free posets [Fis70], upper-triangular matrices with nonnegative integer entries of total sum $n$ and no zero rows or columns [DP10], [BLR14], regular linearized chord diagrams or Stoimenow matchings [Sto98], ascent sequences [BMCDK10], or non-2-neighbor-nesting matchings [Lev13]. The generating function (1) is due to D. Zagier [Zag01], who used it to derive an asymptotic expansion for the Fishburn numbers. On the other hand, striking arithmetic

*Email: astraub@illinois.edu 
properties of these numbers, such as a connection with (mock) modular forms [Zag01], [BOPR12] and congruences resembling those of the partition function [AS14], [Gar14] have recently been discovered. This note is concerned with generalizing these congruences.

In order to state the congruences discovered by G. Andrews and J. Sellers, define, as in [AS14], the sets

$$
S(p)=\left\{j \in\{0,1, \ldots, p-1\}: \quad j \equiv \frac{1}{2} n(3 n-1) \text { for some } n\right\}
$$

of residues modulo $p$ which are pentagonal numbers. The main result of [AS14] is the following.

Theorem 1.1. (Andrews and Sellers, [AS14]) Let $p$ be a prime. If $j \in$ $\{1,2, \ldots, p-1-\max S(p)\}$, then

$$
\xi(p m-j) \equiv 0 \quad(\bmod p)
$$

for all positive integers $m$.

For primes $p$, which are quadratic nonresidues modulo 23 , the set $\{1,2, \ldots, p-$ $1-\max S(p)\}$ is nonempty; thus congruences of the form (2) exist for infinitely many primes (indeed, half of them). F. Garvan proved [Gar14] that such congruences also exist for $p=23$ (in which case $p-1-\max S(p)=0$ ) by replacing the set $S(p)$ in Theorem 1.1 with the smaller set $S^{*}(p)=S(p) \backslash\left\{i_{0}\right\}$, where $i_{0} \in\{0,1, \ldots, p-1\}$ is such that $i_{0} \equiv-1 / 24$ modulo $p$. The purpose of this note is to extend all of these congruences to prime powers, thus answering a question posed by Andrews and Sellers [AS14]. The following is a corollary of our main result, which is stated as Theorem 1.5 below.

Theorem 1.2. Let $p \geqslant 5$ be a prime. If $j \in\left\{1,2, \ldots, p-1-\max S^{*}(p)\right\}$, then

$$
\xi\left(p^{\lambda} m-j\right) \equiv 0 \quad\left(\bmod p^{\lambda}\right)
$$

for all positive integers $m$ and $\lambda$.

Example 1.3. Theorem 1.2 includes the congruences

$$
\begin{array}{rlll}
\xi\left(5^{\lambda} m-1\right) \equiv \xi\left(5^{\lambda} m-2\right) & \equiv 0 & \left(\bmod 5^{\lambda}\right), \\
\xi\left(7^{\lambda} m-1\right) & \equiv 0 \quad\left(\bmod 7^{\lambda}\right), \\
\xi\left(11^{\lambda} m-1\right) \equiv \xi\left(11^{\lambda} m-2\right) \equiv \xi\left(11^{\lambda} m-3\right) & \equiv 0 \quad\left(\bmod 11^{\lambda}\right), \\
\xi\left(17^{\lambda} m-1\right) & \equiv 0 \quad\left(\bmod 17^{\lambda}\right), \\
\xi\left(19^{\lambda} m-1\right) \equiv \xi\left(19^{\lambda} m-2\right) & \equiv 0 & \left(\bmod 19^{\lambda}\right),
\end{array}
$$

which generalize the ones obtained in [AS14], as well as

$$
\xi\left(23^{\lambda} m-j\right) \equiv 0 \quad\left(\bmod 23^{\lambda}\right)
$$

for $j \in\{1,2, \ldots, 5\}$, which generalize the additional congruences of [Gar14]. 
The congruences for the Fishburn numbers $\xi(n)$ provided by Theorem 1.2 are a special case of a more general result concerning the numbers $\xi_{r, s}(n)$ defined by

$$
\sum_{n \geqslant 0} \xi_{r, s}(n) q^{n}=(1-q)^{s} \sum_{n \geqslant 0} \prod_{j=1}^{n}\left(1-(1-q)^{r j}\right)=(1-q)^{s} F\left((1-q)^{r}\right),
$$

where $F(q)$ is defined in (8). Clearly, the case $r=1, s=0$ reduces to the Fishburn numbers $\xi(n)=\xi_{1,0}(n)$. The case $r=-1$ has combinatorial significance as well. The numbers $(-1)^{n} \xi_{-1,0}(n)$ count, for instance, upper-triangular matrices with 0-1-entries of total sum $n$ and no zero rows or columns [AJ14], [BLR14]. In the case $s=0$, we obtain the $r$-Fishburn numbers $\xi_{r}(n)=\xi_{r, 0}(n)$ introduced by Garvan [Gar14]. Note that, for positive integers $s$, the numbers

$$
\xi_{r, s}(n)=\sum_{j=0}^{s}\left(\begin{array}{l}
s \\
j
\end{array}\right)(-1)^{j} \xi_{r}(n-j)
$$

are linear combinations of $r$-Fishburn numbers. As mentioned in [Gar14], congruences satisfied by such combinations, such as

$$
\xi(5 m+2)-2 \xi(5 m+1) \equiv 0 \quad(\bmod 5),
$$

have been first observed by S. Garthwaite and R. Rhoades. In order to state the main result of [Gar14], let us introduce the sets

$$
S(p, r, s)=\left\{j \in\{0,1, \ldots, p-1\}: \quad j-s \equiv \frac{1}{2} r n(3 n-1) \text { for some } n\right\}
$$

as well as, for $p \geqslant 5$, the smaller sets $S^{*}(p, r, s)=S(p, r, s) \backslash\left\{i_{0}\right\}$, where $i_{0} \in$ $\{1,2, \ldots, p-1\}$ is such that $i_{0}-s \equiv-r / 24$ modulo $p$. In other words, modulo $p$, the sets $S^{*}(p, r, s) \equiv r S^{*}(p)+s$ are linear translations of the sets $S^{*}(p)$.

Theorem 1.4. (Garvan, [Gar14]) Let $p \geqslant 5$ be a prime, $r$ an integer such that $p \nmid r$, and $s \in\{0,1, \ldots, p-1\}$. If $j \in\left\{1,2, \ldots, p-1-\max S^{*}(p, r, s)\right\}$, then

$$
\xi_{r, s}(p m-j) \equiv 0 \quad(\bmod p)
$$

for all positive integers $m$.

Our main result generalizes these congruences to prime powers. However, as is illustrated by Examples 1.8 and 1.9 below, not all of these congruences extend to congruences modulo $p^{\lambda}$, so that some restriction on $p, r$ and $s$ is necessary. Recall that every rational number $n$ has a unique $p$-adic expansion

$$
n=\sum_{k=\nu_{p}(n)}^{\infty} n_{k} p^{k}
$$

where $n_{k} \in\{0,1, \ldots, p-1\}$ and $\nu_{p}(n)$ is the $p$-adic valuation of $n$. We write $\operatorname{digit}_{k}(n ; p)=n_{k}$. The following is our main result. 
Theorem 1.5. Let $p$ be a prime, and $r, s$ integers such that $p \nmid r$. If $j \in$ $\{1,2, \ldots, p-1-\max S(p, r, s)\}$, then

$$
\xi_{r, s}\left(p^{\lambda} m-j\right) \equiv 0 \quad\left(\bmod p^{\lambda}\right)
$$

for all positive integers $m$ and $\lambda$. Moreover, if $p \geqslant 5$ and the triple $(p, r, s)$ satisfies

$$
\operatorname{digit}_{1}(s-r / 24 ; p) \neq p-1 \text {, }
$$

then the set $S(p, r, s)$ may be replaced with $S^{*}(p, r, s)$.

Note that, if $r=1$ and $s=0$, then condition (7) is satisfied for all primes $p \geqslant 5$, as can be seen from $\left(p^{2}-1\right) / 24 \in \mathbb{Z}$ and

$$
\operatorname{digit}_{1}(-1 / 24 ; p)=\left\lfloor\frac{p^{2}-1}{24 p}\right\rfloor=\left\lfloor\frac{p}{24}\right\rfloor<p-1 .
$$

Hence, Theorem 1.2 is a corollary of Theorem 1.5.

Our proof of Theorem 1.5 is given in Section 3. It relies crucially on results of Andrews, Sellers and Garvan, which are recalled in Section 2, as well as careful applications of Kummer's theorem on $p$-adic valuations of binomial coefficients. For most of the remainder of this introduction, we illustrate Theorem 1.5 with a number of examples.

Example 1.6. In the case $p=5$ and $r=1$, Theorem 1.5 shows that, in addition to

$$
\xi\left(5^{\lambda} m-1\right) \equiv \xi\left(5^{\lambda} m-2\right) \equiv 0 \quad\left(\bmod 5^{\lambda}\right),
$$

which were already observed in Example 1.3, we have the congruences

$$
\xi_{1,3}\left(5^{\lambda} m-1\right) \equiv 0 \quad\left(\bmod 5^{\lambda}\right) .
$$

Combining these with $\xi_{1,3}(n)=\xi(n)-3 \xi(n-1)+3 \xi(n-2)-\xi(n-3)$, we conclude that

$$
\xi\left(5^{\lambda} m-3\right)-2 \xi\left(5^{\lambda} m-4\right) \equiv 0 \quad\left(\bmod 5^{\lambda}\right),
$$

generalizing the congruence (4), which was observed by Garthwaite and Rhoades.

Example 1.7. We have already seen that, if $r=1$ and $s=0$, then the condition (7) in Theorem 1.5 is automatically satisfied for all primes $p \geqslant 5$. Let us illustrate in this example that condition (7) is similarly vacuous for other "small" values of $r$ and $s$. For instance, in the particularly interesting case $s=0$, we find that, if $r \in\{1,2, \ldots, 23\}$, then the only tuples $(p, r, s)$, for which failure of condition (7) prevents additional congruences, are $(5,23,0)$ and $(7,23,0)$. These two cases are discussed in the next example, which demonstrates that the respective congruences indeed fail to extend to prime powers.

To see that in the remaining cases, for which $r \in\{1,2, \ldots, 23\}$ and $s=0$, we can ignore condition (7) in Theorem 1.5, observe that, modulo $p^{2}$,

$$
s-r / 24 \equiv r \frac{p^{2}-1}{24}
$$


and that the right-hand is an integer in $\left\{0,1, \ldots, p^{2}-1\right\}$. Condition (7) is therefore equivalent to the inequality $r\left(p^{2}-1\right) / 24<p(p-1)$. Rewriting this as $(24-r) p>r$, we find that this inequality holds whenever $p>23 \geqslant r$. Checking the remaining small primes individually for all $r \in\{1,2, \ldots, 23\}$, we conclude that the only cases, for which condition (7) fails and max $S(p, r, s)>$ $\max S^{*}(p, r, s)$, are the two cases $(5,23,0)$ and $(7,23,0)$ mentioned above.

A similar analysis can be carried out for other small values of $s$.

Example 1.8. Note that $S(5,23,0)=\{0,1,3\}$ and $S^{*}(5,23,0)=\{0,1\}$, as well as $S(7,23,0)=\{0,2,3,4\}$ and $S^{*}(7,23,0)=\{0,2,3\}$. Hence, by Garvan's Theorem 1.4, the congruences

$$
\xi_{23}(5 m-j) \equiv 0 \quad(\bmod 5), \quad \xi_{23}(7 m-j) \equiv 0 \quad(\bmod 7)
$$

hold for all positive integers $m$ if $j \in\{1,2,3\}$. On the other hand, since condition (7) is not satisfied, Theorem 1.5 shows that of these six families of congruences only three extend to prime powers; namely,

$\xi_{23}\left(5^{\lambda} m-1\right) \equiv 0 \quad\left(\bmod 5^{\lambda}\right), \quad \xi_{23}\left(7^{\lambda} m-2\right) \equiv \xi_{23}\left(7^{\lambda} m-1\right) \equiv 0 \quad\left(\bmod 7^{\lambda}\right)$.

On the other hand, the other three families cannot be extend to prime powers, as is demonstrated by

$$
\xi_{23}\left(5^{2}-3\right) \equiv 10 \not \equiv 0 \quad\left(\bmod 5^{2}\right), \quad \xi_{23}\left(5^{2}-2\right) \equiv 5 \not \equiv 0 \quad\left(\bmod 5^{2}\right),
$$

and $\xi_{23}\left(7^{2}-3\right) \equiv 42 \not \equiv 0$ modulo $7^{2}$. We should mention that these numbers are already quite large. For instance,

$$
\xi_{23}\left(5^{2}-3\right)=105368264798040017097834938676731639668933422960 .
$$

Example 1.9. As mentioned above, the case $r=-1$ has combinatorial significance as well. Following [Gar14], we observe that 1 is a pentagonal number, which implies $p-1 \in S(p,-1,0)$. In fact, for all primes $p>5, p-1 \in$ $S^{*}(p,-1,0)$, so that no congruences are implied by Garvan's Theorem 1.4 for these values of $p, r, s$. In the case $p=5$, on the other hand, $S(5,-1,0)=\{0,3,4\}$ while $S^{*}(5,-1,0)=\{0,3\}$. Theorem 1.4 therefore yields the congruences

$$
\xi_{-1}(5 m-1) \equiv 0 \quad(\bmod 5),
$$

which were conjectured in [AS14]. However, condition (7) is not satisfied for $(p, r, s)=(5,-1,0)$ since $\operatorname{digit}_{1}(1 / 24 ; 5)=4$, so that Theorem 1.5 does not provide an extension of this congruence to prime powers $5^{\lambda}$. Indeed, the congruences do not extend, as is demonstrated by

$$
\xi_{-1}\left(5^{2}-1\right)=11115833059268126770 \equiv 20 \not \equiv 0 \quad\left(\bmod 5^{2}\right) .
$$

This is another illustration that, for $\lambda>1$, we cannot, in general, replace the set $S(p, r, s)$ with the set $S^{*}(p, r, s)$ in Theorem 1.5. 
On the other hand, we note that $S^{*}(5,-1,2)=\{0,2\}$ and that condition (7) is satisfied for $(p, r, s)=(5,-1,2)$. Hence, for $j \in\{1,2\}$ and all positive integers $m$ and $\lambda, \xi_{-1,2}\left(5^{\lambda} m-j\right) \equiv 0$ modulo $5^{\lambda}$, or, equivalently,

$$
\begin{aligned}
& \xi_{-1}\left(5^{\lambda} m-1\right)-2 \xi_{-1}\left(5^{\lambda} m-2\right)+\xi_{-1}\left(5^{\lambda} m-3\right) \equiv 0 \quad\left(\bmod 5^{\lambda}\right), \\
& \xi_{-1}\left(5^{\lambda} m-2\right)-2 \xi_{-1}\left(5^{\lambda} m-3\right)+\xi_{-1}\left(5^{\lambda} m-4\right) \equiv 0 \quad\left(\bmod 5^{\lambda}\right) .
\end{aligned}
$$

Finally, we complement the congruences of Theorem 1.5 with additional congruences for the case when $p$ divides $r$. These congruences are considerably simpler to prove and are provided here only for the sake of completeness and context.

Lemma 1.10. Let $p$ be a prime, and $r$ a nonzero integer. If $j$ is coprime to $p$, then

$$
\xi_{p^{\lambda} r}(p m-j) \equiv 0 \quad\left(\bmod p^{\lambda}\right)
$$

for all positive integers $m$ and $\lambda$.

\section{Preliminaries}

The formal generating series (1) of the Fishburn numbers equals $F(1-q)$, where

$$
F(q)=\sum_{n \geqslant 0}(q ; q)_{n}
$$

is "Kontsevich's strange function" [Zag01]. Note that the series $F(q)$ is peculiar in that it does not converge in any open subset of the complex plane; it is, however, well-defined, since it terminates, whenever $q$ is a root of unity. As in [AS14], we consider the truncated series

$$
F(q, N)=\sum_{n=0}^{N}(q ; q)_{n},
$$

which we dissect as

$$
F(q, N)=\sum_{i=0}^{p-1} q^{i} A_{p}\left(N, i, q^{p}\right),
$$

where $A_{p}(N, i, q)$ is a polynomial in $q$. We record the following crucial divisibility property of these polynomials, which was proved in [AS14].

Lemma 2.1. Let $p$ be a prime. If $i \notin S(p)$, then

$$
A_{p}(p n-1, i, q)=(1-q)^{n} \alpha_{p}(n, i, q),
$$

where the $\alpha_{p}(n, i, q)$ are polynomials in $q$ with integer coefficients. 
As observed in [AS14], numerical evidence suggests that, for $i \notin S(p)$, the polynomials $A_{p}(p n-1, i, q)$ are in fact divisible by $(q ; q)_{n}$.

A similar result is shown in [Gar14] for indices $i$ such that $i \equiv-1 / 24$ modulo a prime coprime to 24 . Since, modulo $p$, one obtains the conclusion of Lemma 2.1, this result allowed Garvan to prove the additional congruences provided by Theorem 1.4.

Lemma 2.2. Let $p \geqslant 5$ and $i \in\{1,2, \ldots, p-1\}$. If $i \equiv-1 / 24$ modulo $p$, then

$$
A_{p}(p n-1, i, q)=\left(\frac{12}{p}\right) p q^{\lfloor p / 24\rfloor} F\left(q^{p}, p n-1\right)+(1-q)^{n} \beta_{p}(n, i, q),
$$

where the $\beta_{p}(n, i, q)$ are polynomials in $q$ with integer coefficients.

\section{Proofs}

Before turning to the proof of Theorem 1.5, we prove some lemmas, which will be helpful for establishing the desired congruences modulo $p^{\lambda}$. In the sequel, given formal power series $A(q), B(q) \in \mathbb{Z}[[q]]$, we write

$$
A(q) \equiv B(q) \quad\left(\bmod p^{\lambda}\right)
$$

to mean that the coefficients of $A(q)$ and $B(q)$ agree modulo $p^{\lambda}$.

To begin with, we recall a well-known result of Kummer [Kum52], which gives the $p$-adic valuation of binomial coefficients. As usual, and as in (6), the $p$-adic digits $n_{0}, n_{1}, n_{2}, \ldots$ of a (possibly negative) integer $n$ are determined by $n_{i} \in\{0,1, \ldots, p-1\}$ and $n=n_{0}+n_{1} p+n_{2} p^{2}+\cdots$.

Theorem 3.1. (Kummer, [Kum52]) Let $p$ be a prime, and $n, k$ integers such that $k \geqslant 0$. Then the $p$-adic valuation of the binomial coefficient $\left(\begin{array}{l}n \\ k\end{array}\right)$ is equal to the number of carries when adding $k$ and $n-k$ in base $p$.

Proof. Since this result is commonly only stated for the case $n \geqslant k \geqslant 0$, we assume that this case has been proved and show here only how to use it to deduce the case of general $n$. First, in the case $n \geqslant 0$ and $n<k$, the binomial coefficient is zero, so that its $p$-adic valuation is $\infty$. On the other hand, the $p$-adic addition of $k$ and $n-k<0$ results in infinitely many carries.

Suppose now that $n<0$. Observe that, for large enough $\lambda$,

$$
\nu_{p}\left(\left(\begin{array}{l}
n \\
k
\end{array}\right)\right)=\nu_{p}\left(\left(\begin{array}{c}
p^{\lambda}+n \\
k
\end{array}\right)\right)
$$

and, by Kummer's theorem in the nonnegative case, the right-hand side is the number of carries when adding $k$ and $p^{\lambda}+n-k$ in base $p$. It only remains to observe that this equals the number of carries when adding $k$ and $n-k$. 
Lemma 3.2. Let $p$ be a prime, $r$ an integer, and $j \in\{1,2, \ldots, p-1\}$. If $s$ is an integer such that

$$
\left(\begin{array}{c}
s \\
p^{2}-j
\end{array}\right) \equiv 0 \quad(\bmod p)
$$

then, for any positive integers $m, \lambda$,

$$
\xi_{p^{2} r, s}\left(p^{\lambda} m-j\right) \equiv 0 \quad\left(\bmod p^{\lambda-1}\right) .
$$

Proof. Using Zagier's generating function (1), we have

$$
\sum_{n \geqslant 0} \xi_{p^{2} r, s}(n) q^{n}=(1-q)^{s} \sum_{n \geqslant 0} \prod_{j=1}^{n}\left(1-(1-q)^{p^{2} r j}\right) .
$$

Expanding the product on the right-hand side, this can be written in the form

$$
\sum_{n \geqslant 0} c_{n}(1-q)^{p^{2} r n+s}
$$

for some integer coefficients $c_{n}$. It therefore suffices to show that, for any integer $a$, the coefficient of $q^{p^{\lambda} m-j}$ in $(1-q)^{p^{2} a+s}$ vanishes modulo $p^{\lambda-1}$. Equivalently, it is enough to prove that, for any integer $a$,

$$
\left(\begin{array}{c}
p^{2} a+s \\
p^{\lambda} m-j
\end{array}\right) \equiv 0 \quad\left(\bmod p^{\lambda-1}\right)
$$

By Kummer's Theorem 3.1, the p-adic valuation of the binomial coefficient $\left(\begin{array}{l}n \\ k\end{array}\right)$ is equal to the number of carries when adding $k$ and $n-k$ in base $p$. Write $n=p^{2} a+s, k=p^{\lambda} m-j$ and $k^{\prime}=n-k$. Denote with $k_{0}, k_{1}, \ldots$ the $p$-adic digits of $k=p^{\lambda} m-j$, and, likewise, with $k_{0}^{\prime}, k_{1}^{\prime}, \ldots$ the digits of $k^{\prime}$. By construction, $k_{0}=p-j$ and $k_{1}=k_{2}=\ldots=k_{\lambda-1}=p-1$. Since the digits $k_{1}, k_{2}, \ldots, k_{\lambda-1}$ have maximal value, if a carry occurs when adding $k_{0}+k_{1} p$ and $k_{0}^{\prime}+k_{1}^{\prime} p$, then the number of carries when adding $k$ and $k^{\prime}$ is at least $\lambda-1$. Hence, (11) follows from Kummer's theorem.

It remains to observe that, again by Kummer's Theorem 3.1, assumption (10) is equivalent to a carry occuring when adding $k_{0}+k_{1} p$ and $k_{0}^{\prime}+k_{1}^{\prime} p$.

Lemma 3.3. Let $\lambda \geqslant 1$ be an integer. For integers $r$ and $n$ such that $n \geqslant \lambda$,

$$
\left(1-(1-q)^{r p}\right)^{n} \equiv O\left(q^{p n-(p-1)(\lambda-1)}\right) \quad\left(\bmod p^{\lambda}\right) .
$$

Proof. Note that the case $\lambda=1$ is an immediate consequence of the fact that $(1-q)^{p} \equiv 1-q^{p}$ modulo $p$. In general, we conclude that $(1-q)^{p}=1+p q f(q)-q^{p}$ for some polynomial $f(q) \in \mathbb{Z}[q]$. It follows from another binomial expansion that, for any integer $r$,

$$
1-(1-q)^{r p}=1-\left(1+p q f(q)-q^{p}\right)^{r}=p q h_{1}(q)+q^{p} h_{2}(q)
$$


for some power series $h_{1}(q), h_{2}(q) \in \mathbb{Z}[[q]]$. Therefore, modulo $p^{\lambda}$,

$$
\begin{aligned}
\left(1-(1-q)^{r p}\right)^{n} & =\sum_{k=0}^{n}\left(\begin{array}{l}
n \\
k
\end{array}\right)\left(p q h_{1}(q)\right)^{k}\left(q^{p} h_{2}(q)\right)^{n-k} \\
& \equiv \sum_{k=0}^{\lambda-1}\left(\begin{array}{l}
n \\
k
\end{array}\right)\left(p q h_{1}(q)\right)^{k}\left(q^{p} h_{2}(q)\right)^{n-k} \\
& =O\left(q^{(\lambda-1)+p(n-(\lambda-1))}\right)
\end{aligned}
$$

as claimed.

Lemma 3.4. Let $p$ be a prime, $a$ an integer and $i \in\{0,1, \ldots, p-1\}$. If $j$ is an integer such that $0<j<p-i$, then

$$
\left(\begin{array}{c}
p a+i \\
p^{\lambda} m-j
\end{array}\right) \equiv 0 \quad\left(\bmod p^{\lambda}\right)
$$

Proof. Write $n=p a+i$ and $k=p^{\lambda} m-j$. By Kummer's Theorem 3.1, we need to show that there are at least $\lambda$ carries when adding $k$ and $n-k$ in base $p$. Let $n_{0}, n_{1}, \ldots$ be the $p$-adic digits of $n$, and let $k_{0}, k_{1}, \ldots$ be the $p$-adic digits of $k$. By assumption, $n_{0}=i$ and $k_{0}=p-j$, so that the condition on $j$ implies that $k_{0}>n_{0}$. In other words, a carry occurs when adding the least-order $p$-adic digits of $k$ and $n-k$. Moreover, since $k_{1}=k_{2}=\ldots=k_{\lambda-1}=p-1$, we conclude that adding $k$ and $n-k$ in base $p$ results in at least $\lambda$ carries.

We are now in a position to prove Theorem 1.5.

Proof of Theorem 1.5. We need to show that the coefficient of $q^{p^{\lambda} m-j}$ in

$$
(1-q)^{s} F\left((1-q)^{r}\right)=\lim _{N \rightarrow \infty}(1-q)^{s} F\left((1-q)^{r}, N\right)
$$

vanishes modulo $p^{\lambda}$. Let $n \geqslant \lambda$ be an integer. Following [AS14], we split the truncated series

$$
F\left((1-q)^{r}, p n-1\right)=\sum_{i=0}^{p-1}(1-q)^{r i} A_{p}\left(p n-1, i,(1-q)^{r p}\right)
$$

into the part corresponding to indices $i \in S(p)$ and a second part corresponding to indices $i \notin S(p)$. Here, the polynomials $A_{p}$ are as defined by (9). If $i \notin S(p)$, then Lemma 2.1 implies that

$$
A_{p}\left(p n-1, i,(1-q)^{r p}\right)=\left(1-(1-q)^{r p}\right)^{n} \alpha_{p}\left(n, i,(1-q)^{r p}\right) .
$$

It therefore follows from Lemma 3.3 that, for $i \notin S(p)$,

$$
A_{p}\left(p n-1, i,(1-q)^{r p}\right) \equiv O\left(q^{p n-(p-1)(\lambda-1)}\right) \quad\left(\bmod p^{\lambda}\right)
$$


(In fact, it suffices to observe the simpler fact that $A_{p}\left(p n-1, i,(1-q)^{r p}\right)=$ $O\left(q^{n}\right)$.) We conclude that, modulo $p^{\lambda}$,

$F\left((1-q)^{r}, p n-1\right) \equiv \sum_{i \in S(p)}(1-q)^{r i} A_{p}\left(p n-1, i,(1-q)^{r p}\right)+O\left(q^{p n-(p-1)(\lambda-1)}\right)$.

Choosing $n$ large enough, it therefore suffices to show that the coefficient of $q^{p^{\lambda} m-j}$ in

$$
(1-q)^{r i+s} A_{p}\left(p n-1, i,(1-q)^{r p}\right)
$$

vanishes modulo $p^{\lambda}$ for all $i \in S(p)$.

First, suppose that $j \in\{1,2, \ldots, p-1-\max S(p, r, s)\}$. Since $A_{p}(p n-1, i, q)$ is a polynomial in $q$, it suffices to show that, for any integer $a$,

$$
\left[q^{p^{\lambda} m-j}\right](1-q)^{a p+r i+s} \equiv 0 \quad\left(\bmod p^{\lambda}\right) .
$$

Since $r$ is relatively prime to $p$, the condition $i \in S(p)$ implies $r i+s \equiv i^{\prime}$ modulo $p$ for some $i^{\prime} \in S(p, r, s)$. The condition on $j$ implies that $j<p-i^{\prime}$, and congruence (13) follows from Lemma 3.4.

Finally, suppose that the triple $(p, r, s)$ satisfies condition (7) and that $j \in$ $\left\{1,2, \ldots, p-1-\max S^{*}(p, r, s)\right\}$. Let $i_{0} \in\{0,1, \ldots, p-1\}$ be such that $i_{0} \equiv$ $-1 / 24$ modulo $p$. If we can show that, for $i=i_{0}$, the coefficient of $q^{p^{\lambda} m-j}$ in (12) vanishes modulo $p^{\lambda}$, then it only remains to consider (12) for $i \in S^{*}(p)=$ $S(p) \backslash\left\{i_{0}\right\}$ and the same argument as in the previous case proves our desired congruence.

By Lemma 2.2,

$$
A_{p}\left(p n-1, i_{0}, q\right)=\left(\frac{12}{p}\right) p q^{\lfloor p / 24\rfloor} F\left(q^{p}, p n-1\right)+O\left((1-q)^{n}\right) .
$$

As above, it follows from Lemma 3.3 that, if $n$ is large enough, then we may ignore the term $O\left((1-q)^{n}\right)$. It therefore suffices to show that the coefficient of $q^{p^{\lambda} m-j}$ in

$$
\left(\frac{12}{p}\right) p(1-q)^{r i_{0}+s+r p\lfloor p / 24\rfloor} F\left((1-q)^{r p^{2}}\right)
$$

vanishes modulo $p^{\lambda}$. Equivalently, we need to prove that

$$
\xi_{p^{2} r, r i_{0}+s+r p\lfloor p / 24\rfloor}\left(p^{\lambda} m-j\right) \equiv 0 \quad\left(\bmod p^{\lambda-1}\right) .
$$

These congruences follow from Lemma 3.2 if

$$
\left(\begin{array}{c}
r i_{0}+s+r p\lfloor p / 24\rfloor \\
p^{2}-j
\end{array}\right) \equiv 0 \quad(\bmod p) .
$$

Observe that the $p$-adic expansion of $-1 / 24$ is $i_{0}+i_{1} p+i_{2} p^{2}+\cdots$, where $i_{1}=\lfloor p / 24\rfloor$. This is easily seen from

$\operatorname{digit}_{1}\left(-\frac{1}{24} ; p\right)=\operatorname{digit}_{1}\left(\frac{p^{2}-1}{24} ; p\right)=\left(\frac{p^{2}-1}{24}-i_{0}\right) / p=\left\lfloor\frac{p^{2}-1}{24 p}\right\rfloor=\left\lfloor\frac{p}{24}\right\rfloor$. 
Since $p \nmid r$, it follows that

$$
r i_{0}+s+r p\lfloor p / 24\rfloor \equiv s-r / 24 \quad\left(\bmod p^{2}\right) .
$$

Combining (14) and (15), it only remains to prove

$$
\left(\begin{array}{c}
s-r / 24 \\
p^{2}-j
\end{array}\right) \equiv 0 \quad(\bmod p) .
$$

Observing that $\operatorname{digit}_{1}\left(p^{2}-j ; p\right)=p-1$, we find that this congruence follows from Kummer's Theorem 3.1 and the assumption that $\operatorname{digit}_{1}(s-r / 24 ; p)<p-1$. This completes our proof.

Remark 3.5. By Kummer's Theorem 3.1, congruence (16) is equivalent to

$$
\operatorname{digit}_{1}(s-r / 24 ; p)<p-1, \quad \text { or } \quad \operatorname{digit}_{0}(s-r / 24 ; p)<p-j .
$$

The purpose of this remark is to note that the second of these conditions does not hold in the cases which are considered in the course of our proof of Theorem 1.5. In other words, one does not obtain a stronger version of Theorem 1.5 by replacing condition (7) with the weaker condition (16).

Recall that $S^{*}(p, r, s)$ is obtained from $S(p, r, s)$ by removing the element $s_{0} \in\{0,1, \ldots, p-1\}$ which is congruent to $s-r / 24$ modulo $p$. By construction, $s_{0}=\operatorname{digit}_{0}(s-r / 24 ; p)$. Note that replacing $S(p, r, s)$ with $S^{*}(p, r, s)$ in Theorem 1.5 provides additional congruences only if $\max S(p, r, s)=s_{0}$ and the value of $j$ is such that $j>p-1-s_{0}$. In this case, we have $p-j \leqslant s_{0}$, so that the second condition in (17) does not hold.

Finally, let us prove the considerably simpler Lemma 1.10.

Proof of Lemma 1.10. We have to show that $\xi_{p^{\lambda} r}(p m+j) \equiv 0$ modulo $p^{\lambda}$ whenever $j$ is not divisible by $p$. These congruences are equivalent to the fact that, modulo $p^{\lambda}$, the generating series (3) for the numbers $\xi_{p^{\lambda} r}(n)$ can be written as

$$
\sum_{n \geqslant 0} \xi_{p^{\lambda} r}(n) q^{n}=\sum_{n \geqslant 0} \prod_{j=1}^{n}\left(1-(1-q)^{p^{\lambda} r j}\right) \equiv \sum_{n \geqslant 0} c_{n} q^{p n},
$$

for some integers $c_{n}$. It therefore suffices to show that

$$
(1-q)^{p^{\lambda} r j} \equiv h\left(q^{p}\right) \quad\left(\bmod p^{\lambda}\right),
$$

for some power series $h(q) \in \mathbb{Z}[[q]]$. After binomially expanding the left-hand side, this last congruence, follows if we can show that, for any integers $n$ and $k$, such that $k>0$ and $p$ does not divide $k$,

$$
\left(\begin{array}{c}
p^{\lambda} n \\
k
\end{array}\right) \equiv 0 \quad\left(\bmod p^{\lambda}\right) .
$$

Again, this is a direct consequence of Kummer's Theorem 3.1. Since the last $p$-adic digit of $k$ is nonzero by assumption, the $p$-adic addition of $k$ and $p^{\lambda} n-k$ has to involve at least $\lambda$ carries in order for the sum $p^{\lambda} n$ to end in $\lambda$ many zero digits, and (18) follows. 


\section{Conclusion}

We have extended the congruences $\xi(p m-j) \equiv 0$ modulo $p$ of Andrews and Sellers [AS14] as well as Garvan [Gar14] to the case of prime powers. Limited numerical evidence suggests that the congruences for the Fishburn numbers provided by Theorem 1.2 are complete. That is, we expect that every congruence of the form

$$
\xi(\alpha m+\beta) \equiv 0 \quad(\bmod \rho),
$$

which holds for all integers $m$, is implied by Theorem 1.2 together with the Chinese Remainder Theorem. We have numerically verified this conjecture for all $\beta<\alpha \leqslant 100$ and $\rho \leqslant 100$ by checking that no additional congruences exist.

Finally, we echo the problem posed in [Gar14] to investigate congruences for coefficients related to other quantum modular forms, of which $q^{1 / 24} F(q)$, as defined in (8), is an instance [Zag01].

Acknowledgements. I am grateful to Frank Garvan for comments on an earlier version of this paper.

\section{References}

[AJ14] G. E. Andrews and V. Jelínek. On $q$-series identities related to interval orders. European Journal of Combinatorics, 39:178-187, July 2014.

[AS14] G. E. Andrews and J. A. Sellers. Congruences for the Fishburn numbers. Preprint, January 2014. arXiv:1401.5345.

[BLR14] K. Bringmann, Y. Li, and R. C. Rhoades. Asymptotics for the number of row-Fishburn matrices. European Journal of Combinatorics, 41:183-196, October 2014.

[BMCDK10] M. Bousquet-Mélou, A. Claesson, M. Dukes, and S. Kitaev. (2+2)free posets, ascent sequences and pattern avoiding permutations. Journal of Combinatorial Theory, Series A, 117(7):884-909, October 2010 .

[BOPR12] J. Bryson, K. Ono, S. Pitman, and R. C. Rhoades. Unimodal sequences and quantum and mock modular forms. Proceedings of the National Academy of Sciences, 109(40):16063-16067, October 2012.

[DP10] M. Dukes and R. Parviainen. Ascent sequences and upper triangular matrices containing non-negative integers. The Electronic Journal of Combinatorics, 17(1):16, 2010.

[Fis70] P. C. Fishburn. Intransitive indifference with unequal indifference intervals. Journal of Mathematical Psychology, 7(1):144-149, February 1970. 
[Gar14] F. G. Garvan. Congruences and relations for $r$-Fishburn numbers. Preprint, June 2014. arXiv:1406.5611.

[Kum52] E. E. Kummer. Über die Ergänzungssätze zu den allgemeinen Reciprocitätsgesetzen. Journal für die reine und angewandte Mathematik, 44:93-146, 1852.

[Lev13] P. Levande. Fishburn diagrams, Fishburn numbers and their refined generating functions. Journal of Combinatorial Theory, Series A, 120(1):194-217, January 2013.

[Sto98] A. Stoimenow. Enumeration of chord diagrams and an upper bound for Vassiliev invariants. Journal of Knot Theory and its Ramifications, 7(01):93-114, 1998.

[Zag01] D. Zagier. Vassiliev invariants and a strange identity related to the Dedekind eta-function. Topology, 40(5):945-960, September 2001. 